\title{
Implementation of a gauge-invariant time-dependent configuration-interaction-singles method for three-dimensional atoms
}

\author{
Takuma Teramura $\odot,{ }^{1, *}$ Takeshi Sato, ${ }^{1,2,3, \dagger}$ and Kenichi L. Ishikawa ${ }^{1,2,3, \$}$ \\ ${ }^{1}$ Department of Nuclear Engineering and Management, Graduate School of Engineering, University of Tokyo, \\ 7-3-1 Hongo, Bunkyo-ku, Tokyo 113-8656, Japan \\ ${ }^{2}$ Photon Science Center, Graduate School of Engineering, University of Tokyo, 7-3-1 Hongo, Bunkyo-ku, Tokyo 113-8656, Japan \\ ${ }^{3}$ Research Institute for Photon Science and Laser Technology, University of Tokyo, 7-3-1 Hongo, Bunkyo-ku, Tokyo 113-0033, Japan
}

(Received 22 May 2019; published 4 October 2019)

\begin{abstract}
We present a numerical implementation of the gauge-invariant time-dependent configuration-interactionsingles (TDCIS) method [Appl. Sci 8, 433 (2018)] for three-dimensional atoms. In our implementation, an orbital-like quantity called a channel orbital [Phys. Rev. A 74, 043420 (2006)] is propagated instead of configuration-interaction (CI) coefficients, which removes a computational bottleneck of explicitly calculating and storing numerous virtual orbitals. Furthermore, besides its physical consistency, the gauge-invariant formulation allows us to take advantage of the velocity gauge treatment of the laser-electron interaction over the length gauge one in the simulation of high-field phenomena. We apply the present implementation to high-order harmonic generation from helium and neon atoms, which numerically confirms the gauge invariance and demonstrates the effectiveness of the rotated velocity gauge treatment.
\end{abstract}

DOI: 10.1103/PhysRevA.100.043402

\section{INTRODUCTION}

Recent laser technology capable of generating strong laser pulses with an intensity $\gtrsim 10^{14} \mathrm{~W} / \mathrm{cm}^{2}$ has enabled us to explore electron dynamics in the nonperturbative regime, e.g., high-order harmonic generation (HHG), above threshold ionization, nonsequential double ionization, and attosecond pulse generation [1-3]. While laser-driven electron dynamics is rigorously described by the time-dependent Schrödinger equation (TDSE), its direct numerical solution is practically unfeasible for systems with more than two electrons. For theoretical investigation of multielectron dynamics in intense laser field, various tractable $a b$ initio methods have been developed, e.g., time-dependent multiconfiguration self-consistent field (TD-MCSCF) methods [4-13], time-dependent coupled cluster method [14,15], time-dependent $R$-matrix approach [16-20], and time-dependent reduced two-body density matrix approach $[21,22]$.

Among them, the time-dependent configuration-interaction-singles (TDCIS) method is one of promising methods [23-33]. This method has been successfully applied to various electron dynamics such as giant enhancement in HHG in $\mathrm{Xe}$ [32] and decoherence in attosecond photoionization [24]. In the TDCIS method, the total electronic wave function is

\footnotetext{
*teramura@atto.t.u-tokyo.ac.jp

†sato@atto.t.u-tokyo.ac.jp

†ishiken@n.t.u-tokyo.ac.jp
}

Published by the American Physical Society under the terms of the Creative Commons Attribution 4.0 International license. Further distribution of this work must maintain attribution to the author(s) and the published article's title, journal citation, and DOI. approximated by a superposition of time-independent Slater determinants

$$
|\Psi(t)\rangle=\left|\Phi_{0}\right\rangle C_{0}(t)+\sum_{i}^{\mathrm{occ}} \sum_{a}^{\mathrm{vir}}\left|\Phi_{i}^{a}\right\rangle C_{i}^{a}(t),
$$

where $\left|\Phi_{0}\right\rangle$ is the Hartree-Fock (HF) ground state and $\left|\Phi_{i}^{a}\right\rangle$ is a singly excited configuration replacing an occupied orbital $\phi_{i}$ with a virtual orbital $\phi_{a}$ unoccupied in the ground state. The orbital functions are fixed and propagation of configurationinteraction (CI) coefficients $\left(C_{0}\right.$ and $\left.\left\{C_{i}^{a}\right\}\right)$ describes the system dynamics. Although applications of the TDCIS method are limited to the single excitation or ionization due to the truncation of CI space, its low computational cost and ease of analysis are attractive.

The conventional TDCIS method with CI coefficients has two major issues: the explicit calculation and storage of virtual orbitals $\left\{\phi_{a}\right\}$ and a violation of gauge invariance. Virtual orbitals $\left\{\phi_{a}\right\}$ should include both bound and continuum orbitals, whose number is infinite in principle. In a practical simulation with real-space grids, one has to prepare virtual orbitals in advance by numerically obtaining all eigenstates of the discretized HF equation. The number of the virtual orbitals increases with the number of the grid points. Thus, the calculation and storage of virtual orbitals become unacceptably demanding for molecules with a large number of grid points. To solve this problem, Rohringer et al. have proposed an alternative but equivalent formulation of the TDCIS method in which a time-dependent orbital-like quantity called a channel orbital is propagated instead of the CI coefficient [23]. The channel orbital is defined by using $\left\{\phi_{a}\right\}$ and $C_{i}^{a}$ as

$$
\left|\chi_{i}(t)\right\rangle \equiv \sum_{a}^{\mathrm{vir}}\left|\phi_{a}\right\rangle C_{i}^{a}(t) .
$$


The equations of motions (EOMs) of CI coefficient are converted to those of $C_{0}$ and $\left\{\chi_{i}\right\}$. This reformulation removes the computational bottleneck of handling numerous virtual orbitals, while in principle including all the virtual orbitals within a grid space, and has enhanced the utility of the TDCIS method. However, to the best of our knowledge, the applications of the channel orbital-based TDCIS method have been limited to one-dimensional (1D) model in Refs. [23,34,35] and nobles gas atoms with Hartree-Slater potential in Ref. [24].

The TDCIS method, either CI coefficient based or channel orbital based, suffers from a violation of gauge invariance, as a general consequence of the truncation of CI space. Although it is known that the velocity gauge (VG) offers efficient simulations of high-field phenomena, it was impossible to enjoy this advantage within the TDCIS method. To overcome this difficulty, we have recently reported a gauge-invariant reformulation of the TDCIS method [36]. In our reformulation, a rotated velocity gauge ( $\mathrm{rVG})$ transformed from the length gauge (LG) by a unitary operator has been introduced. This unitary transformation ensures the gauge invariance between the LG and rVG, and Ref. [36] numerically confirmed the equivalence of these gauges for a model 1D Hamiltonian.

In this paper, we report a three-dimensional numerical implementation of the gauge-invariant TDCIS method for atoms subject to a linearly polarized laser pulse. We employ a spherical harmonics expansion of orbital functions with the radial coordinate discretized by a finite-element discrete variable representation (FEDVR) [37-40]. We apply the present implementation to HHG from helium and neon atoms and assess the advantage of the rVG over the LG and VG.

This paper proceeds as follows. In Sec. II, we briefly review the TDCIS methods. The numerical implementation of the gauge-invariant TDCIS method to three-dimensional atoms is given in Sec. III. We describe numerical applications in Sec. IV and conclude this work in Sec. V. We use Hartree atomic units (a.u.) throughout the paper unless otherwise stated.

\section{THEORY}

\section{A. The system Hamiltonian and gauge transformation}

We consider an atom with $N$ electrons with a nucleus located at the origin. The time evolution of the $N$-electron wave function $|\Psi(t)\rangle$ is governed by the TDSE,

$$
i \frac{\partial}{\partial t}|\Psi(t)\rangle=\hat{H}(t)|\Psi(t)\rangle,
$$

where $\hat{H}(t)$ is the time-dependent nonrelativistic Hamiltonian

$$
\hat{H}(t)=\hat{H}_{0}+\hat{H}_{\text {ext }}(t),
$$

decomposed into the field-free part,

$$
\hat{H}_{0}=\sum_{i=1}^{N} \hat{h}_{0}\left(\boldsymbol{r}_{i}, \boldsymbol{p}_{i}\right)+\sum_{i>j}^{N} \frac{1}{\left|\boldsymbol{r}_{i}-\boldsymbol{r}_{j}\right|}
$$

and the laser-electron interaction part

$$
\hat{H}_{\mathrm{ext}}(t)=\sum_{i=1}^{N} \hat{h}_{\mathrm{ext}}\left(\boldsymbol{r}_{i}, \boldsymbol{p}_{i}, t\right)
$$

In these expressions, $\boldsymbol{r}_{i}$ and $\boldsymbol{p}_{i}=-i \nabla_{i}$ are the position and the canonical momentum of the electron $i$, respectively. $\hat{h}_{0}$ is given by

$$
\hat{h}_{0}(\boldsymbol{r}, \boldsymbol{p})=\frac{\boldsymbol{p}^{2}}{2}-\frac{Z}{|\boldsymbol{r}|},
$$

where $Z$ is the atomic number. Within the electric dipole approximation, $\hat{h}_{\text {ext }}$ for the LG and VG are given by

$$
\begin{aligned}
& \hat{h}_{\mathrm{ext}}^{\mathrm{LG}}(\boldsymbol{r}, \boldsymbol{p}, t)=\boldsymbol{E}(t) \cdot \boldsymbol{r}, \\
& \hat{h}_{\mathrm{ext}}^{\mathrm{VG}}(\boldsymbol{r}, \boldsymbol{p}, t)=\boldsymbol{A}(t) \cdot \boldsymbol{p},
\end{aligned}
$$

where $\boldsymbol{E}(t)$ and $\boldsymbol{A}(t)=-\int_{-\infty}^{t} d t^{\prime} \boldsymbol{E}\left(t^{\prime}\right)$ are the electric field and the vector potential of the external laser field, respectively.

The two gauges are physically equivalent, and any physical observable takes the same value, independent of the choice of the gauge. The LG wave function $\left|\Psi^{\mathrm{LG}}\right\rangle$ and $\mathrm{VG}$ wave function $\left|\Psi^{\mathrm{VG}}(t)\right\rangle$ are mutually transformed by a gauge transformation as

$$
\begin{gathered}
\left|\Psi^{\mathrm{VG}}(t)\right\rangle=\hat{U}(t)\left|\Psi^{\mathrm{LG}}(t)\right\rangle \\
\hat{U}(t) \equiv \exp \left[-i \sum_{i=1}^{N}\left(\boldsymbol{A}(t) \cdot \boldsymbol{r}_{i}-\frac{1}{2} \int_{-\infty}^{t} d t^{\prime}\left|\boldsymbol{A}\left(t^{\prime}\right)\right|^{2}\right)\right] .
\end{gathered}
$$

\section{B. The CI coefficient-based TDCIS method in the length gauge}

In the conventional TDCIS method based on CI coefficients, orbitals satisfy the canonical, restricted HF equation

$\hat{f}\left|\phi_{p}\right\rangle \equiv \hat{h}_{0}\left|\phi_{p}\right\rangle+\sum_{i}^{\text {occ }}\left(2 \hat{W}_{\phi_{i}}^{\phi_{i}}\left|\phi_{p}\right\rangle-\hat{W}_{\phi_{p}}^{\phi_{i}}\left|\phi_{i}\right\rangle\right)=\epsilon_{p}\left|\phi_{p}\right\rangle$,

where $\hat{f}$ is the Fock operator and $\hat{W}_{\phi^{\prime}}^{\phi}$ is the potential due to the product of two given orbitals $\phi$ and $\phi^{\prime}$, defined in the real space as

$$
\hat{W}_{\phi^{\prime}}^{\phi}\left(\boldsymbol{r}_{1}\right) \equiv \int d \boldsymbol{r}_{2} \frac{\phi^{*}\left(\boldsymbol{r}_{2}\right) \phi^{\prime}\left(\boldsymbol{r}_{2}\right)}{\left|\boldsymbol{r}_{1}-\boldsymbol{r}_{2}\right|} .
$$

$\epsilon_{p}$ is the orbital energy of orbital $\phi_{p}$. In the TDCIS wave function in Eq. (1), $\left|\Phi_{0}\right\rangle$ is the HF ground state formed with the occupied orbitals as

$$
\left|\Phi_{0}\right\rangle=\prod_{i}^{\text {occ }} \hat{c}_{i \uparrow}^{\dagger} \hat{c}_{i \downarrow}^{\dagger}|\rangle
$$

where $\hat{c}_{p \sigma}^{\dagger}$ and $\hat{c}_{p \sigma}$ are the creation and annihilation operators, respectively, of spin-orbital $\phi_{p} \otimes \sigma$, and |\rangle is the vacuum. $\sigma \in\{\uparrow, \downarrow\}$ denotes the spin function. $\left|\Phi_{i}^{a}\right\rangle$ is a singly excited configuration replacing an occupied orbital $\phi_{i}$ with a virtual orbital $\phi_{a}$

$$
\left|\Phi_{i}^{a}\right\rangle=\frac{1}{\sqrt{2}}\left(\hat{c}_{a \uparrow}^{\dagger} \hat{c}_{i \uparrow}+\hat{c}_{a \downarrow}^{\dagger} \hat{c}_{i \downarrow}\right)\left|\Phi_{0}\right\rangle .
$$

The EOMs of CI coefficients is derived through the DiracFrenkel time-dependent variational principle [41], requiring the Lagrangian $L(t)$

$$
L(t)=\left\langle\Psi\left|\hat{H}(t)-i \frac{\partial}{\partial t}-E_{0}\right| \Psi\right\rangle
$$

to be stationary with respect to the variation of $C_{0}^{*}$ and $\left\{C_{i}^{a *}\right\}$. $E_{0}=\left\langle\Phi_{0}\left|\hat{H}_{0}\right| \Phi_{0}\right\rangle$ denotes the HF energy. This constant shift, 
introduced for the simple notation of the EOMs, does not affect physical results. In the LG case, in which the wave function is written as

$$
\left|\Psi^{\mathrm{LG}}(t)\right\rangle=\left|\Phi_{0}\right\rangle C_{0}+\sum_{i}^{\mathrm{occ}} \sum_{a}^{\mathrm{vir}}\left|\Phi_{i}^{a}\right\rangle C_{i}^{a},
$$

the EOMs of CI coefficients are obtained as

$$
\begin{aligned}
i \dot{C}_{0}= & \sqrt{2} \boldsymbol{E} \cdot \sum_{j}^{\mathrm{occ}} \sum_{b}^{\mathrm{vir}}\left\langle\phi_{j}|\boldsymbol{r}| \phi_{b}\right\rangle C_{j}^{b} \\
i \dot{C}_{i}^{a}= & \left\langle\phi _ { a } \left|\left\{\sum_{j}^{\mathrm{occ}} \sum_{b}^{\mathrm{vir}} \hat{F}_{i j}\left|\phi_{b}\right\rangle C_{j}^{b}+\sum_{b}^{\mathrm{vir}} \boldsymbol{E} \cdot \boldsymbol{r}\left|\phi_{b}\right\rangle C_{i}^{b}\right.\right.\right. \\
& \left.+\sqrt{2} \boldsymbol{E} \cdot \boldsymbol{r}\left|\phi_{i}\right\rangle C_{0}\right\}-\boldsymbol{E} \cdot \sum_{j}^{\mathrm{occ}}\left\langle\phi_{j}|\boldsymbol{r}| \phi_{i}\right\rangle C_{j}^{a},
\end{aligned}
$$

where

$$
\begin{gathered}
\hat{F}_{i j}\left|\phi_{b}\right\rangle=\delta_{j}^{i}\left(\hat{f}-\epsilon_{i}\right)\left|\phi_{b}\right\rangle+2 \hat{W}_{\phi_{b}}^{\phi_{j}}\left|\phi_{i}\right\rangle-\hat{W}_{\phi_{i}}^{\phi_{j}}\left|\phi_{b}\right\rangle . \\
\text { C. The channel orbital-based TDCIS method } \\
\text { in the length gauge }
\end{gathered}
$$

\section{The channel orbital-based TDCIS method in the length gauge}

The EOMs of CI coefficients [Eq. (17)] can be rewritten, by substituting channel orbital Eq. (2) into Eq. (17), as

$$
\begin{aligned}
i \dot{C}_{0}= & \sqrt{2} \boldsymbol{E} \cdot \sum_{j}^{\text {occ }}\left\langle\phi_{j}|\boldsymbol{r}| \chi_{j}\right\rangle, \\
i\left|\dot{\chi}_{i}\right\rangle= & \hat{P}\left\{(\hat{F}+\boldsymbol{E} \cdot \boldsymbol{r})\left|\chi_{i}\right\rangle+\sqrt{2} \boldsymbol{E} \cdot \boldsymbol{r}\left|\phi_{i}\right\rangle C_{0}\right\} \\
& -\boldsymbol{E} \cdot \sum_{j}^{\text {occ }}\left|\chi_{j}\right\rangle\left\langle\phi_{j}|\boldsymbol{r}| \phi_{i}\right\rangle,
\end{aligned}
$$

where

$$
\hat{F}\left|\chi_{i}\right\rangle=\left(\hat{f}-\epsilon_{i}\right)\left|\chi_{i}\right\rangle+\sum_{j}^{\mathrm{occ}}\left(2 \hat{W}_{\chi_{j}}^{\phi_{j}}\left|\phi_{i}\right\rangle-\hat{W}_{\phi_{i}}^{\phi_{j}}\left|\chi_{j}\right\rangle\right),
$$

and $\hat{P}$ is the projection operator onto the space spanned by virtual orbitals

$$
\hat{P}=\sum_{a}^{\text {vir }}\left|\phi_{a}\right\rangle\left\langle\phi_{a}\left|=\hat{1}-\sum_{j}^{\text {occ }}\right| \phi_{j}\right\rangle\left\langle\phi_{j}\right|,
$$

with $\hat{1}$ being the identity operator.

\section{Velocity gauge and rotated velocity gauge}

One can, in principle, derive the EOMs for the VG case in the same way as for the LG. Let us write the total wave function and channel orbital as

$$
\begin{gathered}
\left|\Psi^{\mathrm{VG}}(t)\right\rangle=\left|\Phi_{0}\right\rangle D_{0}+\sum_{i}^{\mathrm{occ}} \sum_{a}^{\mathrm{vir}}\left|\Phi_{i}^{a}\right\rangle D_{i}^{a}, \\
|\eta(t)\rangle=\sum_{a}^{\mathrm{vir}}\left|\phi_{a}\right\rangle D_{i}^{a} .
\end{gathered}
$$

$\left|\Phi_{0}\right\rangle$ and $\left|\Phi_{i}^{a}\right\rangle$ are the same configurations as those used in the LG case. Their EOMs are obtained through the same procedures as in the LG case as

$$
\begin{aligned}
i \dot{D}_{0}= & \sqrt{2} \boldsymbol{A} \cdot \sum_{j}^{\text {occ }}\left\langle\phi_{j}|\boldsymbol{p}| \eta_{j}\right\rangle \\
i\left|\dot{\eta}_{i}\right\rangle= & \hat{P}\left\{(\hat{F}+\boldsymbol{A} \cdot \boldsymbol{p})\left|\chi_{i}\right\rangle+\sqrt{2} \boldsymbol{A} \cdot \boldsymbol{p}\left|\phi_{i}\right\rangle C_{0}\right\} \\
& -\boldsymbol{A} \cdot \sum_{j}^{\text {occ }}\left|\eta_{j}\right\rangle\left\langle\phi_{j}|\boldsymbol{p}| \phi_{i}\right\rangle .
\end{aligned}
$$

It is known that TDCIS, which uses time-independent orbitals, is not gauge invariant $[36,42,43]$. Instead of the conventional $\mathrm{VG}$ as described above, we have recently proposed the rVG [36], where we define the rVG wave function by the gauge transformation from the LG wave function as

$$
\left|\Psi^{\mathrm{rVG}}(t)\right\rangle=\hat{U}(t)\left|\Psi^{\mathrm{LG}}(t)\right\rangle .
$$

The rVG orbitals are related to the LG ones by

$$
\begin{gathered}
\left|\phi_{p}^{\prime}(t)\right\rangle=\hat{u}(t)\left|\phi_{p}\right\rangle, \\
\left|\chi_{i}^{\prime}(t)\right\rangle=\hat{u}(t)\left|\chi_{i}\right\rangle=\sum_{a}^{\mathrm{vir}}\left|\phi_{a}^{\prime}\right\rangle C_{i}^{a},
\end{gathered}
$$

where

$$
\hat{u}(t)=\exp \left\{-i\left(\boldsymbol{A}(t) \cdot \boldsymbol{r}-\frac{1}{2} \int_{-\infty}^{t} d t^{\prime}\left|\boldsymbol{A}\left(t^{\prime}\right)\right|^{2}\right)\right\} .
$$

They satisfy the following EOMs [36]:

$$
\begin{aligned}
i \dot{C}_{0}= & \sqrt{2} \boldsymbol{E} \cdot \sum_{j}^{\mathrm{occ}}\left\langle\phi_{j}^{\prime}|\boldsymbol{r}| \chi_{j}^{\prime}\right\rangle, \\
i\left|\dot{\chi}_{i}^{\prime}\right\rangle= & \hat{P}^{\prime}\left\{\left(\hat{F}^{\prime}+\boldsymbol{A} \cdot \boldsymbol{p}\right)\left|\chi_{i}^{\prime}\right\rangle+\sqrt{2} \boldsymbol{E} \cdot \boldsymbol{r}\left|\phi_{i}^{\prime}\right\rangle C_{0}\right\} \\
& -\boldsymbol{E} \cdot \sum_{j}^{\mathrm{occ}}\left(\left|\chi_{j}^{\prime}\right\rangle\left\langle\phi_{j}^{\prime}|\boldsymbol{r}| \phi_{i}^{\prime}\right\rangle+\left|\phi_{j}^{\prime}\right\rangle\left\langle\phi_{j}^{\prime}|\boldsymbol{r}| \chi_{i}^{\prime}\right\rangle\right),
\end{aligned}
$$

where $\hat{P}^{\prime}$ and $\hat{F}_{i}^{\prime}$ are given by Eqs. (21) and (20), respectively, with $\left\{\phi_{j}\right\}$ replaced with $\left\{\phi_{j}^{\prime}\right\}$. Although Eq. (29) contains the dipole operator $\boldsymbol{E} \cdot \boldsymbol{r}$, this does not prevent us from enjoying the advantages of the VG treatment, since it acts only on the localized occupied orbitals $\left\{\phi_{i}^{\prime}\right\}$.

\section{IMPLEMENTATION TO THREE-DIMENSIONAL ATOMS}

\section{A. Spherical-FEDVR basis}

The present implementation is based on our TD-MCSCF code [12], which uses spherical-FEDVR basis functions

$$
\psi_{k l m}(\boldsymbol{r})=\frac{1}{r} \alpha_{k}(r) Y_{l m}(\Omega),
$$

where $r$ and $\Omega$ are the radial and angular coordinate of $\boldsymbol{r}$, respectively, $Y_{l m}$ are spherical harmonics, and $\alpha_{k}$ are radial FEDVR basis functions $[37,38]$. The radial coordinate of the simulation box $\left[0, R_{\max }\right]$ is divided into $K_{\mathrm{FE}}$ finite elements. Each finite element supports $K_{\mathrm{DVR}}$ local DVR functions, and neighboring elements are connected by a bridge function. 
In total, there are $K_{\mathrm{rad}}=K_{\mathrm{FE}} K_{\mathrm{DVR}}-\left(K_{\mathrm{FE}}-1\right)$ radial grid points $\left\{r_{k}\right\}$, on which $\alpha_{k}\left(r_{k^{\prime}}\right)=\delta_{k k^{\prime}} / \sqrt{w_{k}}$, with $\left\{w_{k}\right\}$ being the integration weights.

We expand the channel orbital $\chi_{i}$ in the spherical-FEDVR basis as

$$
\chi_{i}(\boldsymbol{r} ; t)=\sum_{k=2}^{K_{\mathrm{rad}}-1} \sum_{l=0}^{L_{\max }} \psi_{k l m_{i}}(\boldsymbol{r}) g_{i}^{k l}(t),
$$

where $L_{\max }$ is the maximum angular momentum included. The FEDVR basis functions corresponding to $r_{1}=0$ and $r_{K_{\text {rad }}}=$ $R_{\max }$ are removed to enforce the vanishing boundary condition for $r \chi_{i}$ at both ends of the simulation box.

The electrostatic potentials for electron-electron interaction, $\hat{W}_{\phi_{i}}^{\phi_{j}}(\boldsymbol{r})$ and $W_{\chi_{i}}^{\phi_{j}}(\boldsymbol{r}, t)$ required for the EOM of channel orbitals, are computed by solving Poisson's equation,

$$
\begin{aligned}
\nabla^{2} \hat{W}_{\phi_{i}}^{\phi_{j}}(\boldsymbol{r}) & =-4 \pi \phi_{j}^{*}(\boldsymbol{r}) \phi_{i}(\boldsymbol{r}), \\
\nabla^{2} \hat{W}_{\chi_{i}}^{\phi_{j}}(\boldsymbol{r}, t) & =-4 \pi \phi_{j}^{*}(\boldsymbol{r}) \chi_{i}(\boldsymbol{r}, t),
\end{aligned}
$$

using the method described in Ref. [12]. It should be noted that $\hat{W}_{\phi_{i}}^{\phi_{j}}(\boldsymbol{r})$ is time independent, and Eq. (32a) needs to be solved only once before the simulation. On the other hand, $\hat{W}_{\chi_{i}}^{\phi_{j}}(\boldsymbol{r}, t)$ is time dependent and should be computed at every time step. However, since its source $\phi_{j}^{*}(\boldsymbol{r}) \chi_{i}(\boldsymbol{r}, t)$ [see Eq. (32b)] and operand $\left\{\phi_{j}(\boldsymbol{r})\right\}$ [see Eq. (20)] are both localized around the atom due to the locality of occupied orbitals, Eq. (32b) can be solved with less computational cost than the similar equation appearing, e.g., in the timedependent Hartree-Fock and TD-MCSCF method [12].

\section{B. Time propagation with exponential time differencing fourth-order Runge-Kutta scheme}

For an efficient propagation of the EOM of channel-orbitalbased TDCIS, we use the exponential time differencing fourth-order Runge-Kutta scheme (ETDRK4) by Krogstad [44-46]. To this end, we arrange $C_{0}$ and $\left\{\chi_{i}\right\}$ into a unified vector $\chi=\left(C_{0}, \chi\right)^{T}$ and rewrite the EOMs of $C_{0}$ and $\left\{\chi_{i}\right\}$ by a matrix form

$$
i \frac{\partial}{\partial t} \boldsymbol{\chi}=\boldsymbol{h} \chi+\boldsymbol{W}[\boldsymbol{\chi}, t]
$$

where $\boldsymbol{h}$ is a chosen stiff part of the right-hand side of the EOM (see below) and $\boldsymbol{W}[\chi, t]$ is a nonstiff remainder. We choose the stiff part $\boldsymbol{h}$ to be either (i) the field-free one-electron Hamiltonian $\hat{h}_{0}$ or (ii) the totality of the oneelectron Hamiltonian $\hat{h}_{0}+\hat{h}_{\text {ext }}(t)$. For the first case (i) with time-independent $\boldsymbol{h}$, the time propagation from $\chi_{n}=\chi\left(t_{n}\right)$ to $\chi_{n+1}=\chi\left(t_{n}+\Delta t\right)$ is given by

$$
\begin{aligned}
\chi_{n+1}= & \varphi_{0}(-i \boldsymbol{h} \Delta t) \chi_{n}-i \Delta t\left[f_{0}(-i \boldsymbol{h} \Delta t) \boldsymbol{W}_{n}\right. \\
& \left.+f_{1}(-i \boldsymbol{h} \Delta t)\left(\boldsymbol{W}_{a}+\boldsymbol{W}_{b}\right)+f_{2}(-i \boldsymbol{h} \Delta t) \boldsymbol{W}_{c}\right],
\end{aligned}
$$

where $f_{1}, f_{2}$, and $f_{3}$ are defined as

$$
\begin{aligned}
& f_{0}(z)=\varphi_{1}(z)-3 \varphi_{2}(z)+4 \varphi_{3}(z), \\
& f_{1}(z)=2 \varphi_{2}(z)-4 \varphi_{3}(z), \\
& f_{2}(z)=-\varphi_{2}(z)+4 \varphi_{3}(z),
\end{aligned}
$$

where $z=-i \boldsymbol{h} \Delta t, \varphi_{0}(z)=e^{z}$, and

$$
\varphi_{k+1}(z)=\frac{1}{z}\left(\varphi_{l}(z)-\frac{1}{k !}\right) \quad(k=0,1,2, \ldots) .
$$

$\boldsymbol{W}_{n}, \boldsymbol{W}_{a}, \boldsymbol{W}_{b}$, and $\boldsymbol{W}_{c}$ are given by

$$
\begin{aligned}
& \boldsymbol{W}_{n}=\boldsymbol{W}\left[\chi_{n}, t_{n}\right], \\
& \boldsymbol{W}_{a}=\boldsymbol{W}\left[\boldsymbol{a}_{n}, t_{n}+\Delta t / 2\right], \\
& \boldsymbol{W}_{b}=\boldsymbol{W}\left[\boldsymbol{b}_{n}, t_{n}+\Delta t / 2\right], \\
& \boldsymbol{W}_{c}=\boldsymbol{W}\left[\boldsymbol{c}_{n}, t_{n+1}\right],
\end{aligned}
$$

where $\boldsymbol{a}_{n}, \boldsymbol{b}_{n}$, and $\boldsymbol{c}_{n}$ are intermediate vectors given as

$$
\begin{aligned}
\boldsymbol{a}_{n}= & \varphi_{0}(z / 2) \chi_{n}-i \Delta t \varphi_{1}(z / 2) \boldsymbol{W}_{n} / 2, \\
\boldsymbol{b}_{n}= & \varphi_{0}(z / 2) \chi_{n}-i \Delta t \varphi_{1}(z / 2) \boldsymbol{W}_{n} / 2 \\
& -i \Delta t \varphi_{2}(z / 2)\left(\boldsymbol{W}_{a}-\boldsymbol{W}_{n}\right), \\
\boldsymbol{c}_{n}= & \varphi_{0}(z) \chi_{n}-i \Delta t \varphi_{1}(z) \boldsymbol{W}_{n}-2 i \Delta t \varphi_{2}(z)\left(\boldsymbol{W}_{b}-\boldsymbol{W}_{n}\right) .
\end{aligned}
$$

The operator exponential $\varphi_{0}(z)$ and $\varphi_{0}(z / 2)$ in the sphericalFEDVR basis are approximated by the Padé (3/3) approximation, and higher order $\varphi_{k}$ functions are obtained by successively applying Eq. (36). The denominator of the Padé approximation is factorized and operated by the matrix iteration method [12]. We follow Ref. [45] for the modification required for a time-dependent stiff part $\boldsymbol{h}$. In the absence of a linear part for $C_{0}$, time propagation of $C_{0}$ reduces to the well-known fourth-order Runge-Kutta scheme.

\section{Expectation value}

The expectation value of one-body operator $\langle O\rangle=$ $\langle\Psi|O| \Psi\rangle$ can be evaluated in the LG case as $[23,36]$

$$
\langle O\rangle=\sum_{i}^{\mathrm{occ}}\left\{2\left\langle\phi_{i}|O| \phi_{i}\right\rangle+\left\langle\chi_{i}|O| \chi_{i}\right\rangle\right\}+2 \sqrt{2} \operatorname{Re}\left[C_{0} \sum_{i}^{\mathrm{occ}}\left\langle\chi_{i}|O| \phi_{i}\right\rangle\right]-\sum_{i j}^{\mathrm{occ}}\left\langle\chi_{i} \mid \chi_{j}\right\rangle\left\langle\phi_{j}|O| \phi_{i}\right\rangle .
$$

The VG expression is obtained by simply replacing $\left\{C_{0}, \chi_{j}\right\}$ with $\left\{D_{0}, \eta_{j}\right\}$, and the rVG one by replacing $\left\{\phi_{j}, \chi_{j}\right\}$ with $\left\{\phi_{j}^{\prime}, \chi_{j}^{\prime}\right\}$. The Ehrenfest theorem $\frac{d}{d t}\langle O\rangle=-i\langle\Psi|[O, \hat{H}]| \Psi\rangle$ does not hold for TDCIS. Instead, we evaluate the time derivative of $\langle O\rangle$ as [36]

$$
\langle\dot{O}\rangle \equiv \frac{d\langle O\rangle}{d t}=2 \operatorname{Re}\left[\sum_{i}^{\mathrm{occ}}\left\{\left\langle\chi_{i}|O| \dot{\chi}_{i}\right\rangle+\sqrt{2} \dot{C}_{0}\left\langle\chi_{i}|O| \phi_{i}\right\rangle+\sqrt{2} C_{0}\left\langle\dot{\chi}_{i}|O| \phi_{i}\right\rangle\right]\right]
$$


in the LG case. $\left\{C_{0}, \chi_{j}\right\}$ is to be replaced with $\left\{D_{0}, \eta_{j}\right\}$ for VG. The rVG case needs extra terms [36]:

$$
\begin{aligned}
\langle\dot{O}\rangle= & 2 \operatorname{Re}\left[\sum_{i}^{\mathrm{occ}}\left\{\left\langle\chi_{i}^{\prime}|O| \dot{\chi}_{i}^{\prime}\right\rangle+\sqrt{2} \dot{C}_{0}\left\langle\chi_{i}^{\prime}|O| \phi_{i}^{\prime}\right\rangle+\sqrt{2} C_{0}\left\langle\dot{\chi}_{i}^{\prime}|O| \phi_{i}^{\prime}\right\rangle\right\}-\sum_{i j}^{\mathrm{occ}}\left\langle\chi_{i}^{\prime} \mid \dot{\chi}_{j}^{\prime}\right\rangle\left\langle\phi_{j}^{\prime}|O| \phi_{i}^{\prime}\right\rangle\right] \\
& -\sqrt{2} \operatorname{Im}\left[C_{0} \sum_{i}^{\mathrm{occ}}\left\{2 \boldsymbol{E} \cdot\left\langle\chi_{i}^{\prime}|O \hat{r}| \phi_{i}^{\prime}\right\rangle+|\boldsymbol{A}|^{2}\left\langle\chi_{i}^{\prime}|O| \phi_{i}^{\prime}\right\rangle\right\}\right]-i \boldsymbol{E} \cdot \sum_{i j}^{\mathrm{occ}}\left(2 \delta_{j}^{i}-\left\langle\chi_{i}^{\prime} \mid \chi_{j}^{\prime}\right\rangle\right)\left\langle\phi_{j}^{\prime}|[\hat{r}, O]| \phi_{i}^{\prime}\right\rangle .
\end{aligned}
$$

Equations (39), (40), and (41) are valid not only for atoms but also any multielectron system including molecules.

\section{Ionization probability}

To conveniently analyze how ionization proceeds using the TD-MCSCF wave functions with time-varying orbitals, we have previously introduced [10] a domain-based $n$-fold ionization probability $P_{n}$, defined as a probability to find $n$ electrons in the outer region $|\boldsymbol{r}|>R_{\text {ion }}$ and the other $N-n$ electrons in the inner region $|\boldsymbol{r}|<R_{\text {ion }}$ with a given distance $R_{\text {ion }}$ from the origin. This quantity is gauge invariant even during the pulse, unlike the population of the (field-free) continuum levels $[42,43]$. To apply this approach to the TDCIS method with channel orbitals, it is reasonable to assume that the occupied orbitals $\left\{\phi_{i}\right\}$ are localized inside the inner region, i.e., $\phi_{i}(\boldsymbol{r})=0$ for $|\boldsymbol{r}|>R_{\text {ion }}$. Then, the yield of the neutral species or the survival probability $P_{0}$ is computed as

$$
P_{0}(t)=\left|C_{0}(t)\right|^{2}+\sum_{i}^{\mathrm{occ}}\left\langle\chi_{i} \mid \chi_{i}\right\rangle_{<},
$$

where $\left\langle\chi_{i} \mid \chi_{j}\right\rangle_{<}$is the overlap integral in the inner region

$$
\left\langle\chi_{i} \mid \chi_{j}\right\rangle_{<} \equiv \int_{|\boldsymbol{r}|<R_{\text {ion }}} d \boldsymbol{r} \chi_{i}^{*}(\boldsymbol{r} ; t) \chi_{j}(\boldsymbol{r} ; t) .
$$

Noting that the atom described by a TDCIS wave function is at most singly ionized, we obtain the single-ionization probability as $P_{1}(t)=1-P_{0}(t)$.

\section{NUMERICAL EXAMPLE}

We present numerical applications of the implementation of the reformulated TDCIS method described in the previous section and assess efficiency of the rVG. In all simulations reported below, we assume a laser field linearly polarized along the $z$ axis of the following form:

$$
E(t)=\sqrt{I_{0}} \sin (\omega t) \sin ^{2}\left(\pi \frac{t}{N_{\mathrm{opt}} T}\right) \quad\left(0 \leqslant t \leqslant N_{\mathrm{opt}} T\right),
$$

where $I_{0}$ is the peak intensity, $\omega$ is the central frequency, $T=2 \pi / \omega$ is the period, and $N_{\mathrm{opt}}$ is the total number of optical cycles.

\section{A. Helium}

First, we consider helium atom exposed to a laser pulse with $I_{0}=4.0 \times 10^{14} \mathrm{~W} / \mathrm{cm}^{2}, \lambda=400 \mathrm{~nm}$, and $N_{\mathrm{opt}}=12$. In this condition, an exact numerical solution of the TDSE is available [47,48], from which the expectation value of dipole velocity and dipole acceleration can be calculated by using the
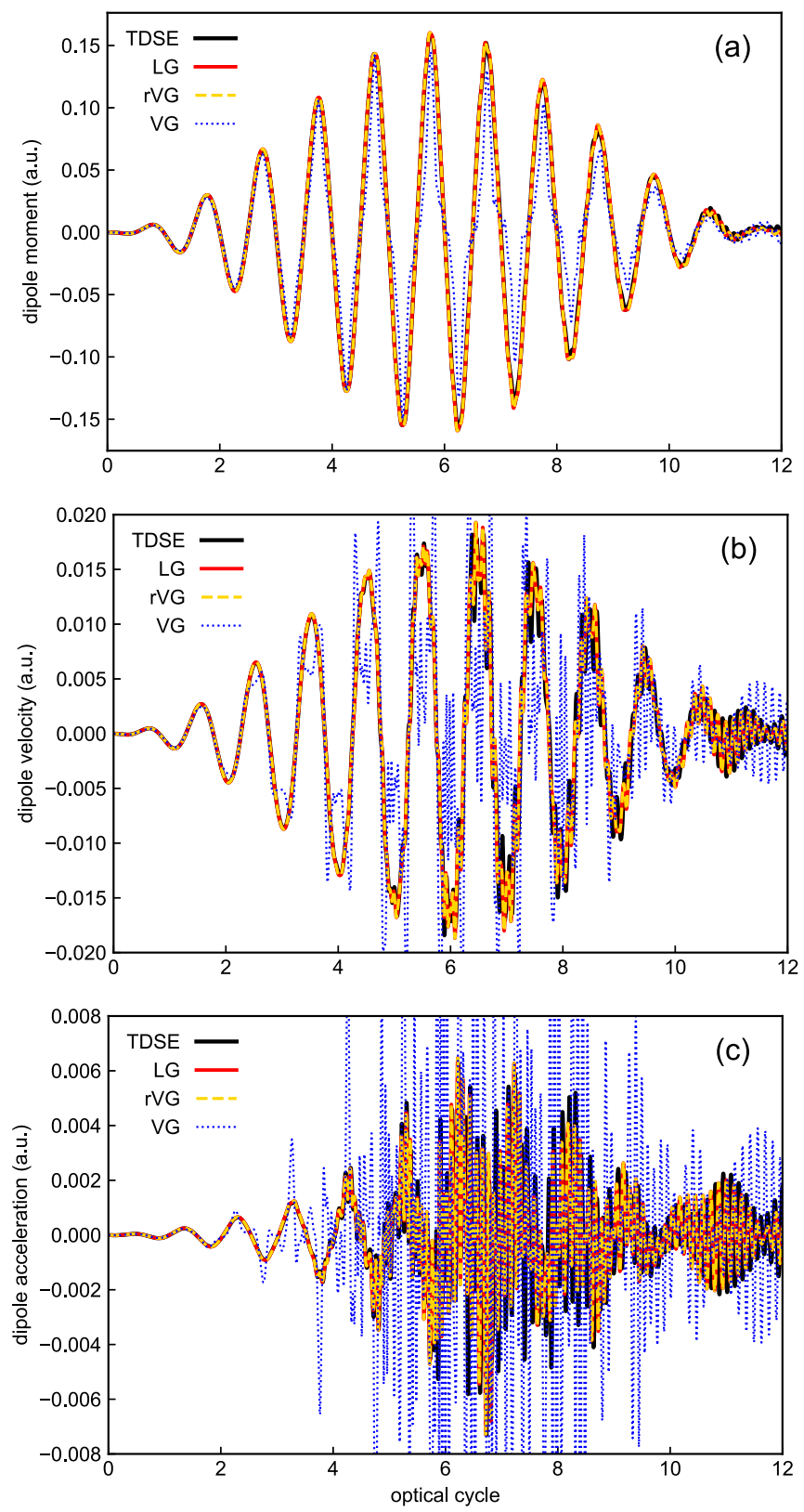

FIG. 1. Time evolution of (a) the dipole moment, (b) the dipole velocity, and (c) the dipole acceleration of $\mathrm{He}$ subject to a laser pulse with $\lambda=400 \mathrm{~nm}, I_{0}=4 \times 10^{14} \mathrm{~W} / \mathrm{cm}^{2}$, and $N_{\text {opt }}=12$, obtained with the exact TDSE (courtesy of Burgdörfer) $[40,47,48]$ and the TDCIS method with length gauge (LG), conventional velocity gauge (VG), and rotated velocity gauge ( $\mathrm{rVG})$. 


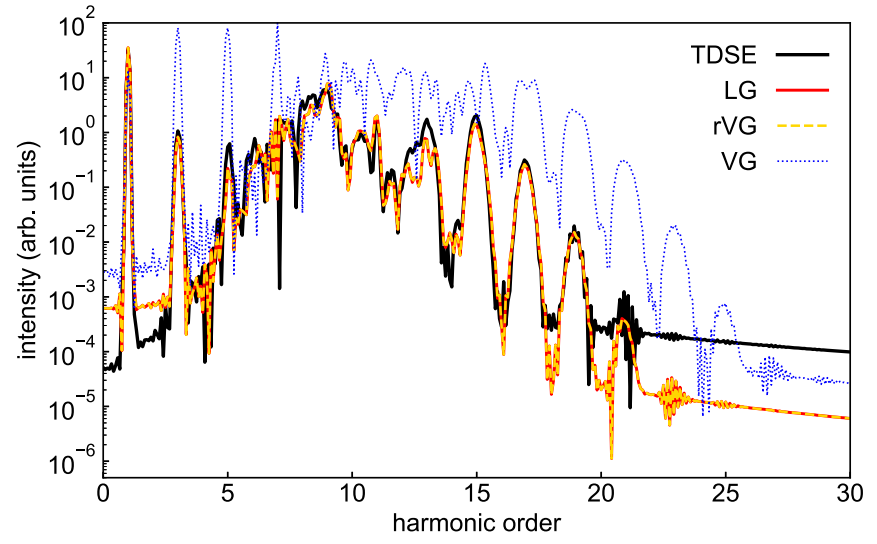

FIG. 2. HHG spectra from He exposed to a laser pulse with the same conditions as Fig. 1, computed from the dipole acceleration shown in Fig. 1(c). Comparison of the TDSE result (courtesy of Burgdörfer) and TDCIS ones with LG, rVG, and VG.

Ehrenfest theorem. For the TDCIS method, we apply $O=\hat{z}$ in Eqs. (39) and (40) to evaluate the expectation value of dipole moment and velocity, respectively. Dipole acceleration is computed by numerically differentiating dipole velocity.

Time evolution of the calculated dipole moment, dipole velocity, and dipole acceleration are shown in Fig. 1, and HHG spectra obtained as the modulus squared of the Fourier transform of the dipole acceleration is presented in Fig. 2. In these figures, one can see the perfect agreement between the LG and rVG results, which numerically confirms the gauge invariance between the two gauges. In contrast, the results of conventional VG with fixed orbitals strongly deviate from them. It should be noted that, from the comparison between LG (and rVG) and VG results alone, we cannot a priori tell which is more accurate. The comparison with the TDSE results now reveals that the former reproduces the TDSE results much better than the latter, which convinces us of an empirical preference of the LG and rVG treatments.

We show the temporal evolution of the survival probability $P_{0}$ with $R_{\text {ion }}=20$ a.u. in Fig. 3 (see the Appendix for the $R_{\text {ion }}$ dependence of $P_{0}$ ). The conventional VG treatment strongly

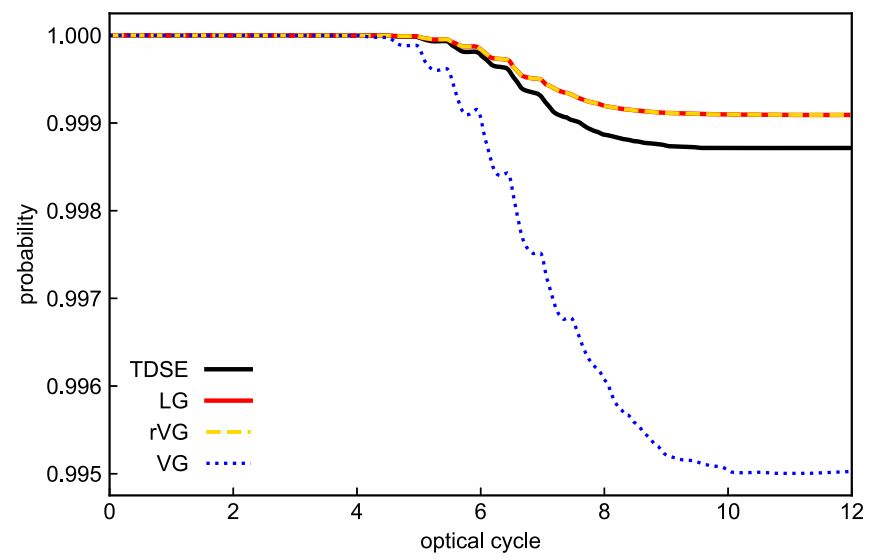

FIG. 3. Time evolution of the survival probability $P_{0}$. Comparison of the TDSE result (courtesy of Burgdörfer) and TDCIS ones with LG, rVG, and VG with $R_{\text {ion }}=20$ a.u.
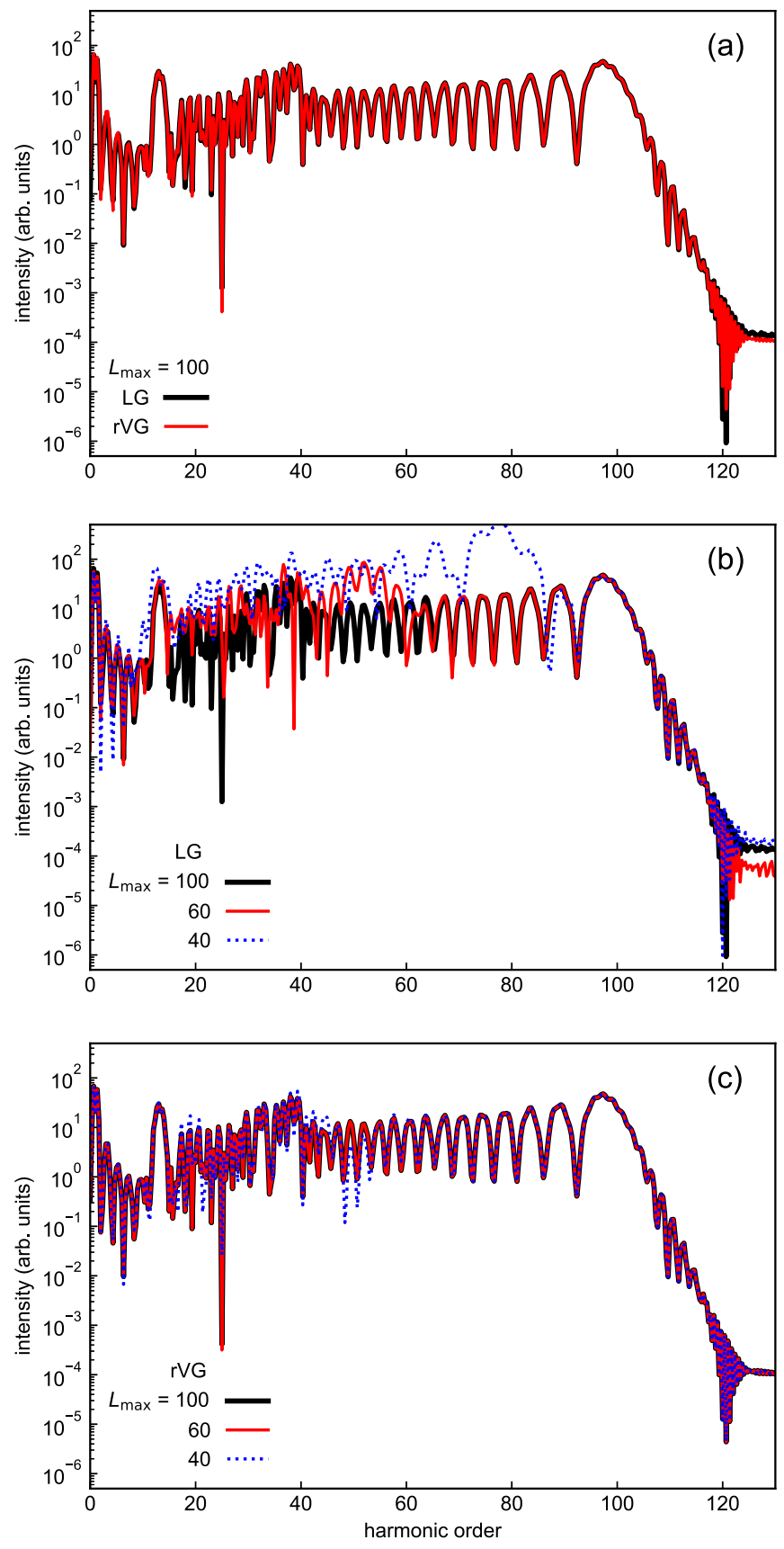

FIG. 4. HHG spectra of Ne subject to an IR laser pulse with a wavelength of $800 \mathrm{~nm}$ and an intensity of $1.0 \times 10^{14} \mathrm{~W} / \mathrm{cm}^{2}$. (a) Results of the LG and rVG with $L_{\max }=100$. (b) Results of the LG with various $L_{\max }$. (c) Results of the rVG with various $L_{\max }$.

overestimates ionization. The fact that tunneling ionization is the first process of the three-step model $[49,50]$ explains the substantial overestimation of the HHG yield in Fig. 2. The LG and rVG results, on the other hand, underestimate tunneling ionization. Correspondingly, we notice that the harmonic intensity is slightly underestimated in Fig. 2.

\section{B. Neon}

We next consider a neon atom subject to a laser field with $\lambda=800 \mathrm{~nm}, I_{0}=8.0 \times 10^{14} \mathrm{~W} / \mathrm{cm}^{2}$, and $N_{\mathrm{opt}}=3$ and 
discuss convergence with respect to the maximum angular momentum $L_{\max }$. We show the HHG spectra calculated with various values of $L_{\max }$ in the LG and rVG in Fig. 4. Figure 4(a) shows the equivalence between the LG and rVG for sufficiently large $L_{\max }(=100)$. As can be seen in Fig. 4(b), which shows $L G$ results, $L_{\max }=60$ is not sufficient to obtain a converged result. On the other hand, the $\mathrm{rVG}$ requires far less $L_{\max }$; even $L_{\max }=40$ well reproduces the result with $L_{\max }=$ 100 , and the spectrum is converged with $L_{\max }=60$ [Fig. 4(c)]. This observation indicates that the rVG TDCIS is simultaneously as accurate as the LG and as efficient as the VG.

\section{CONCLUSIONS}

We have presented a 3D numerical implementation of the recently formulated gauge-invariant TDCIS method [36] for atoms subject to a linearly polarized intense laser field. Compared to the conventional TDCIS method that uses CI coefficients as working variables, the present implementation introduces channel orbitals [23], avoiding calculation and storage of numerous virtual orbitals. We have applied this to $\mathrm{He}$ and $\mathrm{Ne}$ atoms and calculated survival probabilities and HHG spectra for intense laser pulses. The perfect agreement of the LG and $\mathrm{rVG}$ results obtained with a sufficiently large number of partial waves numerically demonstrates the gauge invariance of the method. The comparison with the numerically exact TDSE results for He shows the rVG and LG's superiority to the conventional VG in terms of accuracy. The VG largely overestimates tunneling ionization and then harmonic intensity. The analysis with neon reveals that the rVG has an advantage in computational efficiency over the LG in terms of the number of spherical harmonics required to obtain converged HHG spectrum. Thus, our gauge-invariant reformulation will make TDCIS a promising approach for multielectron dynamics not only in atoms but also in molecules driven by high-intensity laser fields.

\section{ACKNOWLEDGMENTS}

We are grateful to Prof. J. Burgdörfer of Vienna University of Technology for providing us with He TDSE benchmark

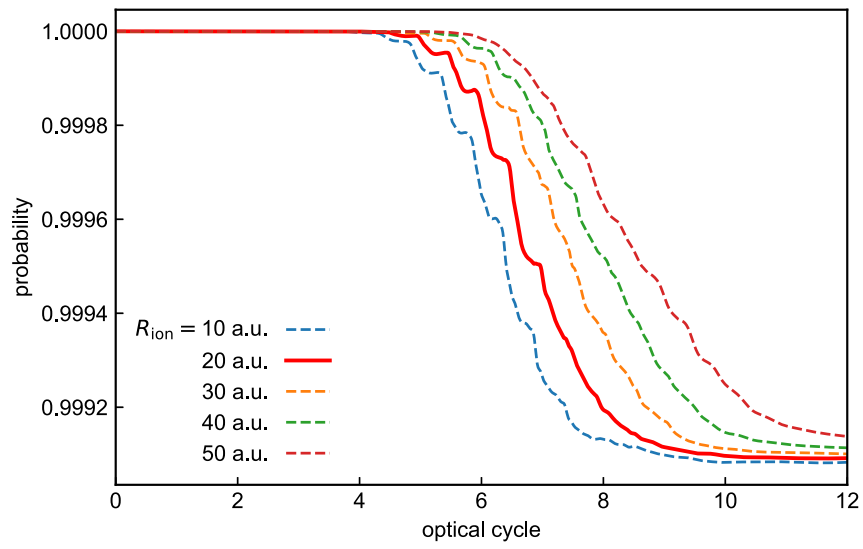

FIG. 5. Temporal evolution of $P_{0}$ for various values of $R_{\text {ion }}$, extracted from LG TDCIS results.

results. This research was supported in part by a Grantin-Aid for Scientific Research (Grants No. 16H03881, No. 17K05070, No. 18H03891, and No. 19H00869) from the Ministry of Education, Culture, Sports, Science and Technology (MEXT) of Japan. This research was also partially supported by JST COI (Grant No. JPMJCE1313), JST CREST (Grant No. JPMJCR15N1), and MEXT Quantum Leap Flagship Program (MEXT Q-LEAP) Grant No. JPMXS0118067246. T.T. gratefully acknowledges support from the Graduate School of Engineering, The University of Tokyo, Doctoral Student Special Incentives Program (SEUT Fellowship).

\section{APPENDIX: $\boldsymbol{R}_{\text {ion }}$ DEPENDENCE ON $\boldsymbol{P}_{0}$}

We show $P_{0}$ from the LG TDCIS results with various values of $R_{\text {ion }}$ in Fig. 5. We can see stepwise ejection of electron wave packets that propagate outward; the larger $R_{\text {ion }}$ is, the later $P_{0}$ is depleted. In the cases of $R_{\text {ion }}=10,20$, and 30 a.u., $P_{0}$ nearly reaches approximately the same final value in the end of the simlation (after twelve optical cycles).
[1] F. Krausz and M. Ivanov, Rev. Mod. Phys. 81, 163 (2009).

[2] M. Nisoli, P. Decleva, F. Calegari, A. Palacios, and F. Martín, Chem. Rev. 117, 10760 (2017).

[3] L. Gallmann, C. Cirelli, and U. Keller, Annu. Rev. Phys. Chem. 63, 447 (2013).

[4] J. Zanghellini, M. Kitzler, C. Fabian, T. Brabec, and A. Scrinzi, Laser Phys. 13, 1064 (2003).

[5] T. Kato and H. Kono, Chem. Phys. Lett. 392, 533 (2004).

[6] D. J. Haxton and C. W. McCurdy, Phys. Rev. A 91, 012509 (2015).

[7] H. Miyagi and L. B. Madsen, Phys. Rev. A 89, 063416 (2014).

[8] J. Caillat, J. Zanghellini, M. Kitzler, O. Koch, W. Kreuzer, and A. Scrinzi, Phys. Rev. A 71, 012712 (2005).

[9] H. Miyagi and L. B. Madsen, Phys. Rev. A 87, 062511 (2013).

[10] T. Sato and K. L. Ishikawa, Phys. Rev. A 88, 023402 (2013).
[11] T. Sato and K. L. Ishikawa, Phys. Rev. A 91, 023417 (2015).

[12] T. Sato, K. L. Ishikawa, I. Březinová, F. Lackner, S. Nagele, and J. Burgdörfer, Phys. Rev. A 94, 023405 (2016).

[13] R. Anzaki, T. Sato, and K. L. Ishikawa, Phys. Chem. Chem. Phys. 19, 22008 (2017).

[14] S. Kvaal, J. Chem. Phys. 136, 194109 (2012).

[15] T. Sato, H. Pathak, Y. Orimo, and K. L. Ishikawa, J. Chem. Phys. 148, 051101 (2018).

[16] M. A. Lysaght, H. W. van der Hart, and P. G. Burke, Phys. Rev. A 79, 053411 (2009).

[17] M. A. Lysaght, P. G. Burke, and H. W. van der Hart, Phys. Rev. Lett. 101, 253001 (2008).

[18] P. G. Burke and V. M. Burke, J. Phys. B 30, L383 (1997).

[19] L. R. Moore, M. A. Lysaght, L. A. Nikolopoulos, J. S. Parker, H. W. Van Der Hart, and K. T. Taylor, J. Mod. Opt. 58, 1132 (2011). 
[20] D. D. A. Clarke, G.S. J. Armstrong, A. C. Brown, and H. W. van der Hart, Phys. Rev. A 98, 053442 (2018).

[21] F. Lackner, I. Březinová, T. Sato, K. L. Ishikawa, and J. Burgdörfer, Phys. Rev. A 91, 023412 (2015).

[22] F. Lackner, I. Březinová, T. Sato, K. L. Ishikawa, and J. Burgdörfer, Phys. Rev. A 95, 033414 (2017).

[23] N. Rohringer, A. Gordon, and R. Santra, Phys. Rev. A 74, 043420 (2006).

[24] A. Gordon, F. X. Kärtner, N. Rohringer, and R. Santra, Phys. Rev. Lett. 96, 223902 (2006).

[25] A. Karamatskou and R. Santra, Phys. Rev. A 95, 013415 (2017).

[26] S. Pabst, L. Greenman, P. J. Ho, D. A. Mazziotti, and R. Santra, Phys. Rev. Lett. 106, 053003 (2011).

[27] L. Greenman, P. J. Ho, S. Pabst, E. Kamarchik, D. A. Mazziotti, and R. Santra, Phys. Rev. A 82, 023406 (2010).

[28] M. Grosser, J. M. Slowik, and R. Santra, Phys. Rev. A 95, 062107 (2017).

[29] S. Pabst and R. Santra, J. Phys. B: At. Mol. Opt. Phys. 47, 124026 (2014).

[30] A. Sytcheva, S. Pabst, S.-K. Son, and R. Santra, Phys. Rev. A 85, 023414 (2012).

[31] S. Pabst, A. Sytcheva, A. Moulet, A. Wirth, E. Goulielmakis, and R. Santra, Phys. Rev. A 86, 063411 (2012).

[32] S. Pabst and R. Santra, Phys. Rev. Lett. 111, 233005 (2013).

[33] E. Heinrich-Josties, S. Pabst, and R. Santra, Phys. Rev. A 89, 043415 (2014).

[34] J. A. You, J. M. Dahlström, and N. Rohringer, Phys. Rev. A 95, 023409 (2017).

[35] J. A. You, N. Rohringer, and J. M. Dahlström, Phys. Rev. A 93, 033413 (2016).

[36] T. Sato, T. Teramura, and K. L. Ishikawa, Appl. Sci 8, 433 (2018).
[37] T. N. Rescigno and C. W. McCurdy, Phys. Rev. A 62, 032706 (2000).

[38] C. W. McCurdy, M. Baertschy, and T. N. Rescigno, J. Phys. B: At. Mol. Opt. Phys. 37, R137 (2004).

[39] B. I. Schneider, L. A. Collins, and S. X. Hu, Phys. Rev. E 73, 036708 (2006).

[40] B. I. Schneider, F. Johannes, S. Nagele, R. Pazourek, S. Hu, L. A. Collins, and J. Burgdörfer, in Quantum Dynamic Imaging, edited by A. D. Bandrauk and M. Ivanov (Springer, New York, 2011), p. 149.

[41] P. Kramer and M. Saraceno, Geometry of the Time-Dependent Variational Principle in Quantum Mechanics (Springer, New York, 1981).

[42] K. L. Ishikawa and T. Sato, IEEE J. Sel. Top. Quantum Electron. 21, 8700916 (2015).

[43] T. Sato, Y. Orimo, T. Teramura, T. Oyunbileg, and K. L. Ishikawa, in Progress in Ultrafast Intense Laser Science XIV, edited by K. Yamanouchi, P. Martin, M. Sentis, L. Ruxin, and D. Normand, Springer Series in Chemical Physics Vol. 118 (Springer International, Cham, 2018), pp. 143-172.

[44] S. Krogstad, J. Comput. Phys. 203, 72 (2005).

[45] M. Hochbruck and A. Ostermann, Acta Num. 19, 209 (2010).

[46] D. Kidd, C. Covington, and K. Varga, Phys. Rev. E 96, 063307 (2017).

[47] J. Feist, S. Nagele, R. Pazourek, E. Persson, B. I. Schneider, L. A. Collins, and J. Burgdörfer, Phys. Rev. Lett. 103, 063002 (2009).

[48] R. Pazourek, J. Feist, S. Nagele, E. Persson, B. I. Schneider, L. A. Collins, and J. Burgdörfer, Phys. Rev. A 83, 053418 (2011).

[49] P. B. Corkum, Phys. Rev. Lett. 71, 1994 (1993).

[50] Super-intense Laser-Atom Physics, edited by B. Piraux, A. L'Huillier, and K. Rzążewski, NATO ASI Series Vol. 316 (Springer US, Boston, MA, 1993), p. 95. 